\title{
Major Hepatic Resection in Hepatic Hydatidosis ${ }^{*}$
}

\author{
Ibrahim Abdelkader Salama ${ }^{1 \#}$, Mohammed Abdallah Aboushady ${ }^{1}$, Osama Hegazy Abdelsalam ${ }^{1}$, \\ Sherif Mohamed Saleh ${ }^{1}$, Hany Abdel Mageed Shoreem ${ }^{1}$, \\ Mohammed Houseni ${ }^{2}$, El-Amir Mahmoud Amir ${ }^{3}$ \\ ${ }^{1}$ Department of Hepatobiliary Surgery, National Liver Institute, Menophyia University, Shiben Elkom, Egypt \\ ${ }^{2}$ Department of Radiology National Liver Institute, Menophyia University, Shiben Elkom, Egypt \\ ${ }^{3}$ Department of Parasitology National Liver Institute, Menophyia University, Shiben Elkom, Egypt \\ Email: \#ibrahim_salama@hotmail.com
}

Received October 14, 2012; revised November 15, 2012; accepted November 23, 2012

\begin{abstract}
Background: Ecchinococcal disease is still a serious problem in certain parts of the world, with liver as the most affected organ. Surgery remains the mainstay of treatment of hydatidosis, but the optimal surgical procedure remains unsettled. Objective: Safety and efficacy of major hepatic resection in multiple and giant hepatic hydatidosis. Patients \& Methods: 63 patients had hepatic hydatidosis associated with spleen, lung, and suprarenal hydatidosis were managed by major hepatic resection between April 2005 to April 2011. 43 (68\%) males and 20 (32\%) females, age range 8 - 56 years. Cysts were found in the liver only in $51(81 \%)$ patients, liver with spleen in $5(8 \%)$, liver, spleen with lung in 2 (3.1\%), liver and lung in $4(6.3 \%)$, liver and suprarenal in one patient (1.6\%). Results: Multiple cysts were found in 38 $(60 \%)$ and solitary cyst in $25(40 \%)$ with Cysts diameter $\leq 5 \mathrm{~cm}$ in $22(35 \%), 5-10 \mathrm{~cm}$ in $16(25 \%)$, and $10-38$ in 25 (40\%). Right hepatectomy in $24(38 \%)$, right trisectionectomy in $2(3.2 \%)$, right hepatectomy with Segment III in 4 $(6.3 \%)$, right hepatectomy with Segment I in $2(3.2 \%)$, left hepatectomy in $12(19 \%)$, left lateral sectionectomy in 6 (9.5\%), left hepatectomy with Segment VI in $3(4.8 \%)$, left lateral sectionectomy with right posterior sectionectomy in 2 $(3.2 \%)$, right hepatectomy with splenectomy in $7(11 \%)$ and right hepatectomy with right suprarenal in one (1.6\%) patient were performed. Hospital stay was 4.2 (3 - 13 days) There was one mortality and 12 (19\%) morbidities. No recurrence on follow up period (8 - 60 months) was observed. Conclusion: Radical procedure is safe and effective option for hepatic hydatidosis and should be performed when the entire lobe is diffusely involved by huge or multiple hydatidosis with little healthy liver tissue.
\end{abstract}

Keywords: Ecchinococcosis; Hepatic Hydatidosis; Hepatic Resection; Mortality; Morbidity

\section{Introduction}

Hydatid disease has been known since the time of Hippocrates and is described as "Liver full of water". The disease is endemic in the Mediterranean region, South America, The Far East, Middle East, and Eastern Europe. However, it is observed more frequently in nonendemic countries being to increasing global travel [1].

Hydatid disease is a zoonosis caused mainly by Echinococcus granulosus, and less frequently by E. multilocularis and E. oligarthrus [2].

The location of the hydatid cysts is mainly hepatic (75\%), pulmonary (15\%) and only $(10 \%)$ of the cysts occur in other parts of the body [3]. Most patients with hydatid cyst of the liver have no symptoms, and its presence becomes evident when the liver is found to be enlarged or a cystic lesion is noted when the liver is im-

\footnotetext{
${ }^{*}$ Conflict of interest: no conflict of interest to declare.

${ }^{\#}$ Corresponding author.
}

aged for other reasons. Large cysts may be painful; however, symptoms may result from a number of complications [3]. In addition, the known complications of the hydatid disease (Rupture, Suppuration and Calcification) and multiplicity of cysts are other common surgical problems $[4,5]$.

Routine laboratory investigations are of little help in diagnosis, and serological tests for hydatidosis are positive in approximately $80 \%$ of patients [6]. Ultrasonographic examination and computed tomography $(\mathrm{CT})$ are very useful in diagnosis and classification of the cysts $[7$, 8].

Surgery remains the mainstay of treatment for hydatid disease and aims to eliminate the parasite promote the rapid disappearance of any residual cavity and prevent complications and recurrence [9-11]. Giant hydatid cyst is life threatening but rare. It has potentially lethal complications, such as anaphylactic shock due to perforation; thus early diagnosis and definitive treatment is life sav- 
ing [12].

When the mortality and morbidity of hepatic resection were high, cystic lesions of the liver were treated conservatively by repeated needle aspiration, fenestration, marsupialization, or deroofing [13,14]. However, hepatic resection has become a safe and effective procedure in the last two decades not only for the malignant, but also for the benign, hepatic lesions $[15,16]$. Radical surgical removal of the hydatid cystic lesion remains the mainstay of treatment with a high success rate [17].

In this study we report our experience with major hepatic resection in Giant and multiple hepatic hydatid cysts, regarding the technique, postoperative morbidity, safety, effectiveness, and recurrence during follow up period.

\section{Patients and Methods}

This study was conducted at Department of Hepatobiliary Surgery, National Liver institute, Menophyia University, Shiben Elkom, Egypt with the approval of scientific and ethical committees during the period from April 2005 to April 2011. Total 63 patients diagnosed with hydatid disease in the liver, some of them had associated hydatid disease in the other organs of the body.

Diagnosis was made by history, clinical examination, indirect haemagglutination inhibition test (Laboratoires Fumouze-division Diagnostics, Paris, France) and enzyme linked immunosorbant assay test for hydatid $\operatorname{IgG}$ (Euroimmun Labordiagnostica, Germany), Abdominal ultrasound/CT, chest, brain CT and MRI in some cases to diagnose associated cysts in the other parts of the body.

The pathognomanic characteristics and ultrasonographic signs of hydatid liver disease have all been included in Gharbi's classification [18] (Table 1).

All patients were treated with Albendazole $(10 \mathrm{mg} / \mathrm{kg})$ 2 weeks before operation, and the medication continued for 2 months postoperatively.

Magnetic Resonance Cholangiopancreatography (MRCP) or Endoscopic Retrograde Cholangiopancreatography (ERCP) were used preoperatively for patients with jaundice, history of cholangitis, dilation of biliary ducts, existence of debris in the bile ducts, or elevation of serum

Table 1. Gharbi’s classification for Hydatid cyst.

\begin{tabular}{cc}
\hline Types of cyst & Description \\
\hline Type I & $\begin{array}{c}\text { Pure (clear) fluid collection } \\
\text { (the cyst is similar to the simple liver cyst) }\end{array}$ \\
Type II & Fluid collection with a split wall (water lily sign) \\
Type III & $\begin{array}{r}\text { Fluid collection with multiple septa/and/or } \\
\text { daugh ter cyst (honey comb sign) }\end{array}$ \\
Type IV & Heterogeneous cyst contents with high internal echoes \\
Type V & Cyst with reflecting calcified thick wall
\end{tabular}

liver transaminases.

Six patients $(9.5 \%)$ referred from other hospital after treated with percutaneous aspiration and then experienced recurrence. Two of them had sign of infection (high fever, leucocytosis, and jaundice) and were operated upon urgently.

\subsection{Surgical Approach}

The types of hepatic resection depend on the size and location of the hydatid cyst of the liver. The abdomen was opened by bilateral subcostal incision with midline extension. After routine abdominal exploration, the liver was mobilized and four pieces of gauze soaked in $20 \%$ hypertonic sodium chloride were introduced into the abdominal cavity and placed around the cyst to prevent the spread and leakage of the hydatid fluid. The intraoperative ultrasound was used to detect the exact site of the cyst or cysts in the liver, and its relation with hepatic vasculature (portal veins, hepatic arteries, and hepatic veins). The peripheral and superficial subcapscular cysts were deactivated by $20 \%$ hypertonic saline solution after aspiration of the hydatid fluid and the saline was left inside the cyst for about 10 minutes to kill the scolices, to prevent accidental spillage of the cyst content and the puncture site was covered with hypertonic saline solution-soaked gauzes.

The liver resection started by dissection of the hilum of the liver and isolation of the right portal triad or left portal triad depending on the site of the liver resection (right or left respectively). In right hepatectomy, the right side hepatic ligament was dissected and the right hepatic vein was isolated and encircled by tape, left side hepatic ligament dissected and middle and left hepatic veins also encircled in the left hepatectomy. Parenchymal dissection was achieved in our cases by Harmonic Scalpel (Harmonic Scalpel, Ethicon EndoSurgery, Cincinnati, Ohio) and Cuvatron Ultrasonic Surgical Aspirator (CUSA) (CUSA, Valley Lab Inc., Boulder, CO). After complete resection the haemostasis was achieved and any minute bile ductules were ligated.

Concomitant splenectomy was performed in 7 cases (11\%) where the spleen is the site of hydatid cyst associated with the liver. Right adrenelectomy was performed with right hepatectomy in one case (1.6\%) as the hydatid cyst infected the right adrenal gland. The abdomen was closed with drains. Lung hydatid cyst was associated in 6 patients (9.5\%), 5 cases treated by lung resection 2 weeks prior to the liver resection, while one patient who had calcified hydatid in the lung was treated conservatively and followed up.

Early postoperative morbidity and hospital course were noted. Postoperative follow up after resection with ultrasonography was performed as follows: 1st postoperative day and before removal of the drains. All patients were followed up by clinical examination, serological 
test, ultrsonography of the abdomen, CT in particular cases, and liver functions. Manifestations such as reappearance of the liver cysts at the site of previously treated cyst or the appearance of new extrahepatic disease resulting from procedure-related spillage were considered as local recurrence.

\subsection{Statistical Analysis}

Statistical analysis was performed using the student $t$ test for postoperative hospital stay and $\times 2$ test for morbidity. $P$ value $\leq 0.05$ was considered significant.

\section{Results}

Forty-three $(68 \%)$ out of sixty-three patients were males and twenty (32\%) were females, with Age range from 8 56 years.

Seventeen patients (27\%) had history of exposure to sheep and/or dogs, 12 patients (19\%) had history of residence abroad (5 patients in Iraq, and 7 patients in Libya). However, no history of exposure or residence abroad was observed in 34 patients (54\%).

The most common symptom was pain in the right upper quadrant in 32 patients $(50.5 \%)$, abdominal mass in the right upper quadrant in 15 patients (24\%), jaundice in 6 patients $(9.5 \%)$, jaundice and fever in 2 patients $(3.5 \%)$ who were presented by acute abdomen operated upon urgently. However, 8 asymptomatic patients (12.5\%) discovered the mass incidentally during routine radiology for other causes.

Cysts were found in the liver only in 51 patients (81\%) (Figure 1), liver and spleen in 5 patients $(8 \%)$ (Figure 2), liver and lung in 4 patients (6.3\%) (Figure 3), liver, lung and spleen in 2 patients (3.1\%) and liver with right suprarenal hydatid cyst in one patient (1.6\%).

Cysts were found in the right lobe of the liver in 34 patients $(54 \%)$, in the left lobe in 18 patients $(28.5 \%)$ and in both lobes in 11 patients (17.5\%).

Thirty-eight patients $(60 \%)$ had multiple hydatid cysts (19 patients in right lobe, 8 patients in left lobe and 11 patients in both lobes) and 25 patients $(40 \%)$ had solitary huge cyst (15 patients in right lobe and 10 patients in left lobe) (Figure 4).

The diameter of the cysts was $5 \mathrm{~cm}$ or less in $22 \mathrm{pa}-$ tients (35\%), $5-10 \mathrm{~cm}$ in 16 patients (25\%) and larger than $10 \mathrm{~cm}$ reach $38 \mathrm{~cm}$ in 25 patients $(40 \%)$. Six patients $(9.5 \%)$ had infected cysts (Table 2).

\subsection{Types of Liver Resection}

The hepatic resections were performed in those patients carrying the hydatid disease depended on the site and location of the lesion. Formal right hepatectomy was carried out in 24 patients (38\%), right trisectionectomy
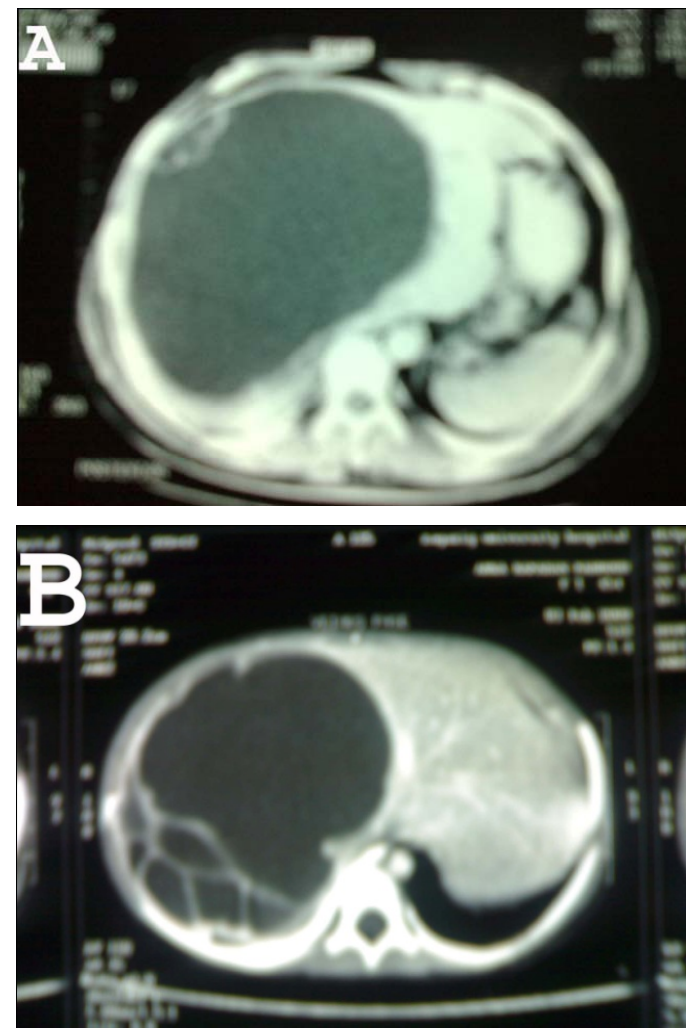

Figure 1. Abdominal CT Scan showing huge right lobe hydatid cyst.
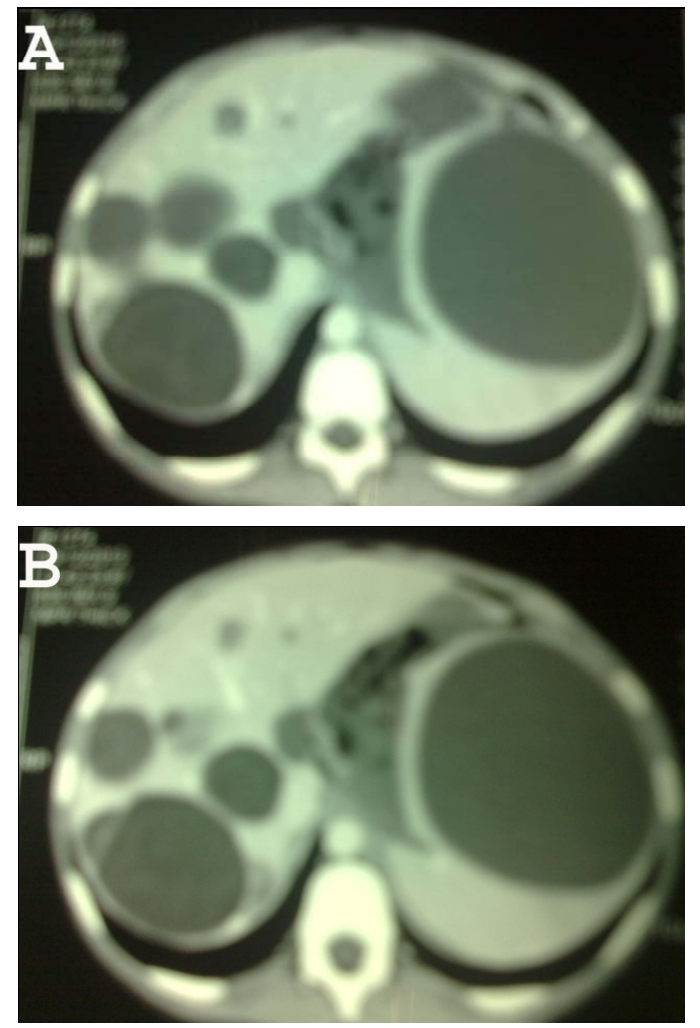

Figure 2. Abdominal CT Scan showing multiple hydatid cysts in both lobe of the liver and in spleen. 

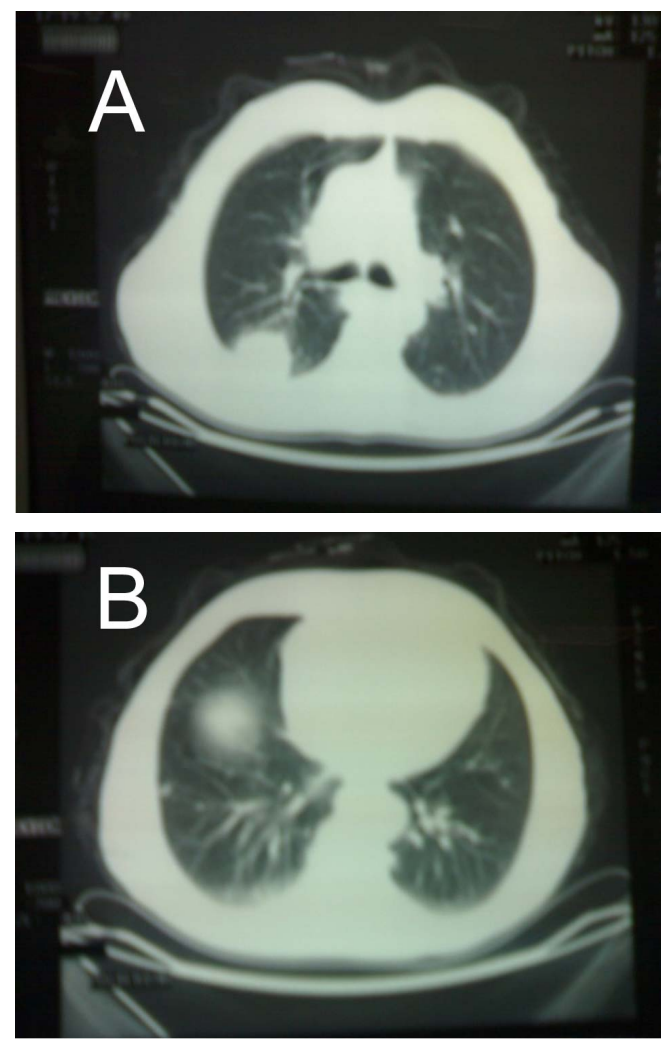

Figure 3. CT of the chest showing hydatid cyst of the lung (A) lower lobe of the right lung; (B) Middle zone of the right.

Table 2. Morphological features and localization of hydatid cysts.

\begin{tabular}{cc}
\hline & No. of patients $=63 \%$ \\
\hline Site of organ & \\
Liver only & $51(81 \%)$ \\
Liver + spleen & $5(8 \%)$ \\
Liver + lung & $4(6.3 \%)$ \\
Liver + spleen + lung & $2(3.1 \%)$ \\
Liver + right suprarenal & $1(1.6 \%)$ \\
Location of the cysts in the liver & \\
Right lobe & $34(54 \%)$ \\
Left lobe & $18(28.5 \%)$ \\
Both lobes & $11(17.5 \%)$ \\
Number of the cysts & \\
Multiple cysts & $38(60 \%)$ \\
Solitary cyst & $25(40 \%)$ \\
$\leq 5$ & \\
Mean diameter of the cysts $(\mathrm{cm})$ & $22(35 \%)$ \\
$>10$ to 38 cm & $16(25 \%)$ \\
Type I & $25(40 \%)$ \\
Type II & \\
Type III & $6(9.5 \%)$ \\
Type IV & $7(11 \%)$ \\
Type V & $39(62 \%)$ \\
Gharbi morphological type of the cysts & $8(12.5 \%)$ \\
\hline
\end{tabular}
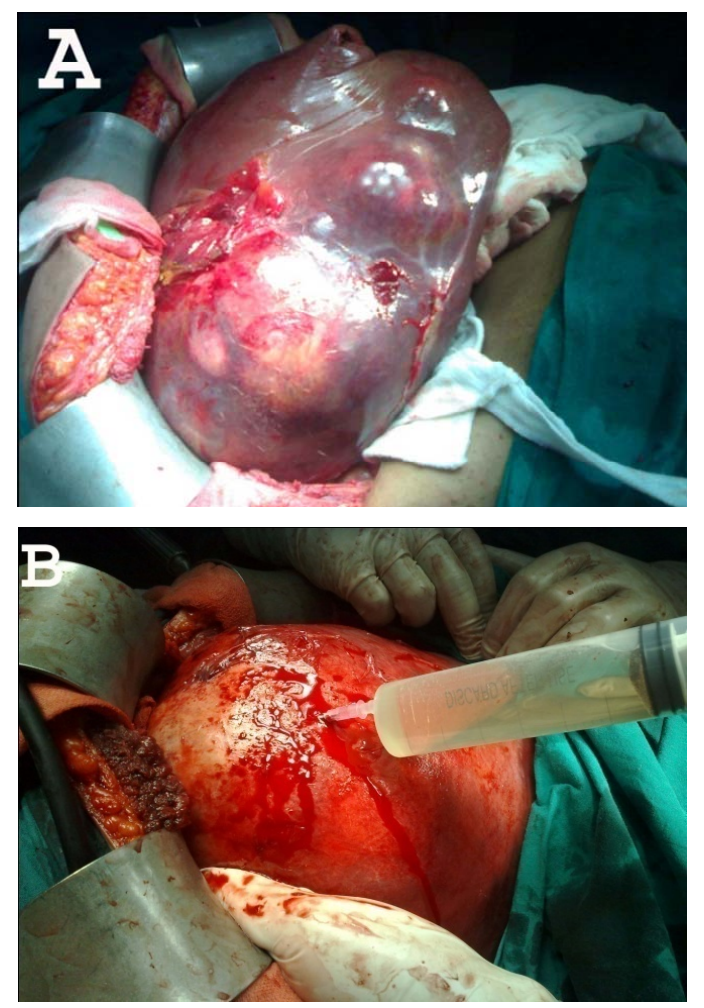

Figure 4. Intraoperative image. (A) Multiple hydatid cysts of the right lobe of the liver; (B) Huge hydatid cyst occupying most of the right lobe of the liver.

was carried out in 2 patients $(3.2 \%)$ in whom the hydatid cysts were multiple occupying the right lobe and segment IV. Right hepatectomy and nonanatomical resection in left lobe were carried out in 6 patients $(9.5 \%) ; 4$ patients (6.3\%) segment III resection and in 2 patients $(3.2 \%)$ segment I resection were done in addition to right hepatectomy). Formal left hepatectomies only were carried out in 12 patients (19\%) and associated with segment VI in 3 patients $(4.8 \%)$. Left lateral sectionectomy only carried out only in 6 patients $(9.5 \%)$ and associated with right posterior sectionectomy in 2 patients $(3.2 \%)$.

Concomitant splenectomies with right hepatectomy were carried out in 7 cases $(11 \%)$ while right adrenalectomy and right hepatectomy were carried out in 1 patient $(1.6 \%)$, (Table 3). In this series there were associated lung hydatid diseases in 6 patients $(9.5 \%), 5$ patients had lung resection two weeks before the hepatectomy and one patient who had small calcified hydatid was treated conservatively and followed up.

\subsection{There Were No Associated Brain Lesions in This Series}

Two patients operated upon urgently as they were referred from other hospital with picture of acute abdomen (fever, jaundice and high white cell count) after percutanous aspiration of multiple cysts in the right lobe. The 
Table 3. Types of major hepatic resection in hydatid disease.

\begin{tabular}{cc}
\hline Type of hepatic resection & No. of patients $=63 \%$ \\
\hline Right hepatectomy & $24(38 \%)$ \\
Right trisectionectomy & $2(3.2 \%)$ \\
Right hepatectomy + segment III resection & $4(6.3 \%)$ \\
Right hepatectomy + Segment I resection & $2(3.2 \%)$ \\
Left hepatectomy & $12(19 \%)$ \\
Left lateral sectionectomy & $6(9.5 \%)$ \\
Left hepatectomy + Segment VI resection & $3(4.8 \%)$ \\
Left lateral sectionectomy + & $2(3.2 \%)$ \\
RT.pos.sectionectomy & $7(11 \%)$ \\
Right hepatectomy + Splenectomy & $1(1.6 \%)$ \\
Right hepatectomy + right adrenalectomy & \\
\hline
\end{tabular}

first patient was 8-year-old girl. On exploration there were multiple right lobe cysts with puncture in 2 cysts. After sterilization and wash out of the abdomen, the right hepatectomy was done. The second patient was 56 years old female. On exploration, there were multiple infected cysts in the right lobe. The right hepatectomy and exploration of the common bile duct and $\mathrm{T}$-tube drainage were done.

Six patients $(9.5 \%)$ had biliary communications diagnosed by ERCP with migration of daughter cysts in CBD in 2 cases in which the preoperative sphinctertomy and retrieval of the daughter cysts from the CBD were successfully achieved. (Figure 5) All those patients underwent choledochotomy followed by irrigation of the ductus choledochus and insertion of T-Tube.

\subsection{Morbidity and Mortality}

There was one mortality in our study, female patient presented with acute abdomen due to rupture hydatid cysts after percutaneous aspiration in another hospital, and she died on the thirteenth day postoperatively due to severe biliary sepsis which leads to multiorgan failure (MOF). Postoperative complications occurred in 12 patients $(19 \%)$, wound infection developed in 6 patients $(9.5 \%)$ treated by meticulous wound care, subphrenic collection in 2 patients $(3.2 \%)$ treated by percutanous drainage and pigtail insertion, biliary fistula occurred in 2 patients $(3.2 \%)$ one treated by aspiration and pigtail drainage and the other treated by papillotomy and insertion of biliary stent. One patient $(1.6 \%)$ who had lung atelectasis was treated by vigorous respirator exercise \& antibiotic according to culture and sensitivity. One patient $(1.6 \%)$ developed incisional hernia after two years postoperatively and was repaired with mesh.

The mean length of hospital stay was 4.2 (range 3 - 13 days).

\subsection{Follow Up}

The patients were followed up by clinical examination,
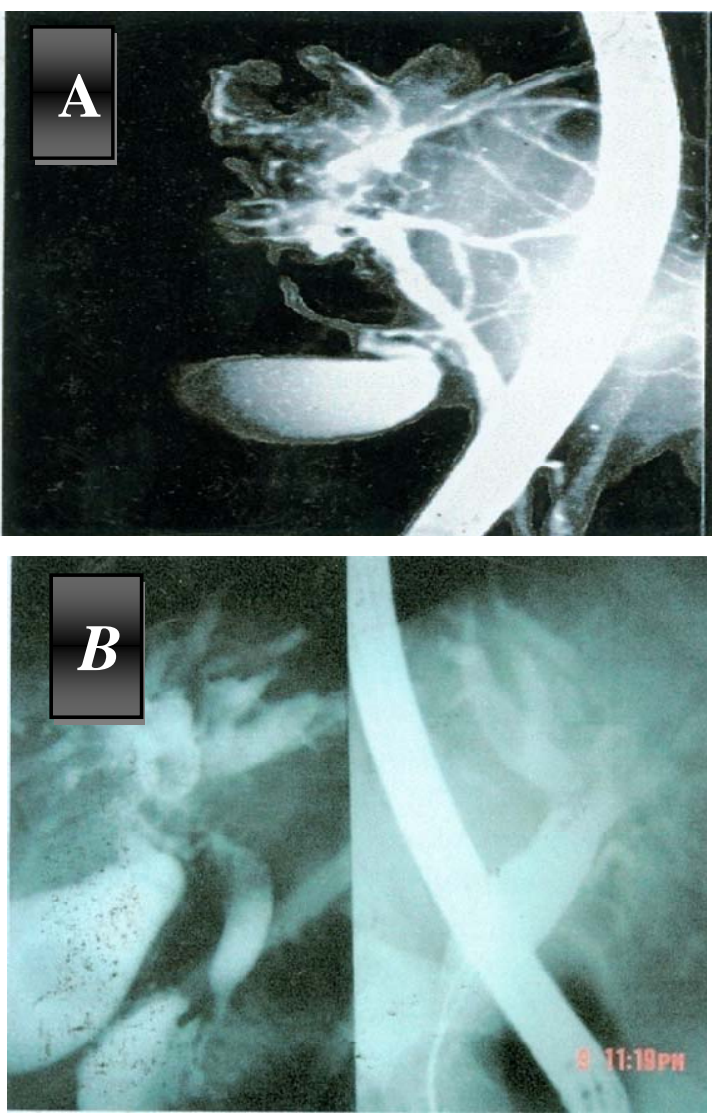

Figure 5. ERCP of hydatid cyst of the liver (A) Showing cystobiliary communication; (B) Showing extraction of cyst contents by dormia basket.

ultrsasonography after I, 3 months postoperatively then every 6 months in first 2 year then annually. In some cases CT was performed for follow up. During the follow up period which ranged from $8-60$ months, there was no evidence of recurrence based on ultrasound and CT images.

\section{Discussion}

In spite of the efforts to control echinococcosis in many parts of the world, the disease is still frequent in endemic areas; the true incidence of this disease is difficult to estimate, as it is usually asymptomatic in most patients [19].

The disease continues to exert a heavy burden on human health in a number of countries, including Egypt and reemerges as a major public health issue, with potentially life-threatening complications [20].

The infection should be suspected in patients who live in rural areas and who present with abdominal pain and hepatomegaly or a palpable hepatic mass [21].

Approximately $50 \%-70 \%$ of the cysts are localized in the liver. The right lobe is affected in $85 \%$ of patients, and in one quarter to one third of cases is multiple [22]. 
The detection of cysts had increased with the advent and routine use of ultrasonography which, in addition to the diagnosis, provides information about the cyst wall, fluid content and surrounding liver tissues. CT is carried out to further delineate the anatomy and rule out proximity of vital structures [23].

Hepatic hydatid cysts are usually single, uncomplicated and located in the right lobe of the liver. A large series of retrospective multicenteric studies for hydatid cyst of the liver indicated that the most frequent clinical patterns were uncomplicated cysts $(82 \%)$; remaining present with complicated cysts [24].

In this study, $12.5 \%$ of patients were asymptomatic while $50.5 \%$ of patients had pain, and $24 \%$ of patients presented by pressure manifestation. In this series, $3.5 \%$ of patients were presented by complication (rupture) due to iatrogenic injuries to the cyst following percutaneous aspiration of the liver hydatid cyst.

The diagnosis of hydatid disease in this study was based primarily upon characteristic feature on U/S and or CT imaging. These features are described in the literatures, [2] with a diagnostic sensitivity of $94 \%-96 \%$ and $100 \%$ respectively, in many reports [25]. The sensitivities of these techniques in this study were $96 \%$ and $100 \%$, respectively.

It has been reported that magnetic resonance imaging (MRI) is very useful in the diagnosis of hydatid disease particularly in the absence of characteristic appearance on U/S or CT imaging and when serologic tests are negative [26]. However, this modality was used in this study in some cases to diagnose the hydatid cyst in the other organs of the body.

Endoscopic retrograde cholangiopancreatography (ERCP) plays an important role in the diagnosis and management particularly in patients with obstructive jaundice or history of jaundice, recurrent cholangitis, or in patients with recurrent hydatid disease. It was done in 11 patients in this study ( 8 with jaundice and 3 with history of jaundice) and revealed cystobiliary communication in 6 patients with migration of daughter cysts in biliary tree in 2 patients. These results are consistent with most of the reports which indicates that ERCP and related therapeutic maneuvers are safe and valuable in the management of patients with hepatic hydatid disease [27,28].

Medical treatment with Benzimidazole derivatives remains disappointing [29]. A multicenter study conducted by the World Health Organization has suggested that the medical treatment should be restricted to inoperable cases or to the prevention of postoperative recurrence [29].

In this series, albendazole was given in a dose of 10 $\mathrm{mg} / \mathrm{kg}$ daily for 2 weeks preoperatively and for 8 weeks after the procedure. This regimen was based on the assumption that at time of surgery any escaping scolices would be maximally exposed to effective tissue and circulating levels of the drug.

Biliary communications are reportedly common in hydatid disease with variable frequencies between 3.5\% [30] and 19\% [31]. Therefore, meticulous attention should be paid to their preoperative detection. Patients who have jaundice or a history of cholangitis, elevated liver enzymes, and dilatation or debris in major bile ducts should be assessed for main bile duct contamination. [10] If the bile ducts are evaluated with ERCP before surgery, it is not necessary to perform main duct exploration. Kayaalp and co-workers have shown that hydatid cysts lying around the hilum of the liver had a higher biliary communication rate $(48 \%)$ than did peripherally located cysts (27\%) [32]. It is still controversial whether a T-tube or biliary enteric anastomosis is advantageous after a duct exploration. Alper and colleagues have shown that choledocoduodenostomy reduces mortality and morbidity rates when compared to the use of a T-tube for drainage [33]. However, Erbir and co-workers compared T-tube drainage with choledochoduodenostomy for mortality and morbidity rates, hospitalization time, and infection rate and showed superiority of the T-tube [34]. In this study, ERCP was done in 11 cases ( 8 had jaundice \& 3 had history of jaundice). In 2 patients ERCP was diagnostic and therapeutic as sphinctertomy \& retrieval of daughter cysts from CBD was successfully achieved. In those patients, T-tube drainage was the treatment of choice.

Surgical methods to obliterate the cyst cavity can be separated as conservative (simple drainage, unroofing, and omentoplasty) or radical procedure (partial cystectomy, pericystectomy and hepatic resections). All these procedures have their own proponents. A tailored approach is required in each patient due to variation in size, multiplicity, location and associated complication. Some authors advocate radical procedures, mainly pericystectomy \& hepatic resection [35].

It is widely accepted that radicality of the intervention increases the operative risk, but lowers the likehood of a relapse and vice versa [36].

Conservative methods are recommended in difficult cases. Some authors believe that radical procedures are time-consuming with increasing blood loss and unjustifiable for a benign disease [37].

Advances in liver surgery have occurred over last two decades, hepatectomy has evolved from a rough, hastily and bloody procedure to a refined, deliberate, and relatively bloodless operation. The preoperative assessment for liver resection from anesthetics point view has advanced with advent of surgical tools (CUSA, Harmonic Scalpel, and Organ beam coagulation) and technique of rendering the hepatic resection become a safe procedure. Intraoperative ultrasound locates the exact size and location of lesion in the liver especially deep seated lesion in 
hepatic parenchyma and its relation with hepatic vasculature. All those improvements permit the performance of precise resections and avoid complications related to inaccurate dissection of the liver parenchyma [38].

In this study, radical surgery was employed in the patients with multiple hydatid cysts or huge hydatid cyst, occupying the entire lobe of the liver, applying major hepatic resection for hydatid disease of the liver which has advantage to do hepatic resection without opening the cyst cavity and therefore avoiding the problems of spillage and cavity management.

Considering the recurrence rates after operation for liver hydatid cysts, it ranges from $8 \%$ - 20\% [39] especially in endemic areas. Multiple operations may be necessary during the course of this benign disease, thus radical operations (hepatectomy) should be performed from the start.

In this study, the multiple cysts of the liver hydatid disease constitute $60 \%$ of the cases. It is advantage to treat the multiple hydatid cysts in one stage, so the hepatectomy can remove all cysts, especially if the cysts are deeply inaccessible position in liver parenchyma. In huge liver hydatid cyst which constitutes $40 \%$ of the cases, it is better to resect the whole liver lobe occupied by the hydatid cyst without opening the cyst for fear of rupture.

In co-existing liver and pulmonary parasites, the lung cysts should be operated either first, because of the greater tendency to rupture and infection, or simultaneously (in cysts of the dome of the liver and right lung), bilateral lung cysts have to be resected in two stages.

In this study, 6 lung cysts were associated with liver hydatid cysts, 5 treated by lung resection 2 weeks before the liver resection, and the other one was small calcified cyst treated conservatively with follow up.

Concomitant splenectomy was carried out in 7 cases with liver resection and right suprarenal was resected with right hepatectomy.

From the literature, it appears that two common problems of major concern following surgery for hepatic hydatid disease are biliary fistula and recurrence.

Postoperative biliary leak or fistula has been reported in a rate of $6 \%-47 \%,[24,35,40]$ as a consequence of small, undetected communications between the cyst and the bile duct. Such communications have been reported to occur in $10 \%-80 \%$ of cases [40].

When conservative procedures are used, the poorly vasculaized pericyst left in situ may disguise biliary communications in the residual cavity. On the contrary, with radical procedure either in the form of liver resection or pericystectomy, the biliary pedicles are more easily identified and safely ligated in the healthy liver parenchyma.

Demirici and coworkers [41] observed a 3.5\% rate of postoperative biliary fistula in 87 patients treated with radical procedures vs $27.5 \%$ in 173 patients treated by conservative procedures. Magistrelli and coworkers [42] observed a higher incidence of biliary leak following conservative rather than radical procedures $(14.1 \%$ vs $6.2 \%$ ). In this study, biliary fistula occurred in 2 patients $(3.5 \%)$ post right hepatectomy treated conservatively with aspiration and papillotomy and stent.

Recurrence after primary treatment of hepatic hydatid disease is an important issue, with reported rates ranging from $1.1 \%$ to $25 \%$ [24,35,43].

Failure to detect pre-existing intrahepatic small cysts during initial operation is the main cause of recurrence. This is now overcome by the use of high resolution imaging diagnostic modalities (U/S and CT) and use of intraoperative ultrasonography. Better understanding of the mechanism of daughter cyst formation as illustrated by Magistrelli has brought attention a new factor implicated in local disease recurrence [42]. Undetected and subsequently untreated exogenous daughter cysts located outside the pericyst may cause local recurrence if a conservative procedure is adopted for the treatment of the main cyst.

In Assadourian [44] series, found 23\% of exogenous daughter cysts. In Magistrelli [42] series, 19\% was noted in 64 patients. In this study we did not find any exogenous cysts as the radical procedures are expected to better control local recurrence of the disease. Moreover, it will eliminate the factor of spillage of hydatid cysts contents, since evacuation of the cyst can be safely omitted in most cases.

Magistrelli and coworkers [42] reported 16\% of local recurrence in patients who underwent conservative procedures and $2 \%$ in 62 patients who underwent radical procedures. In this study, there was no recurrence with a long term follow up period ranging from 8 to 60 months by clinical examination, ulltasongraphy and CT follow up.

Overall postoperative mortality ranges between $0 \%$ to $7.5 \%$ [45-47] in early period. In this study we had one postoperative mortality $(1.6 \%)$ from sepsis of biliary system due to cystobiliary communication which ends to multiorgans failure (MOF).

Morbidity after treatment of hydatid disease of the liver was observed in $12 \%$ - $26 \%$ of cases [46].

Diziri et al. [47] found an overall complication rate of $33 \%$ in their multicentric, prospective, randomized trial. Yorganci and Sayek [35] have reported an even higher complications rate of $40 \%$ which, in their explanation, was due to the presence of a number of complicated cases in their series.

In this study overall postoperative morbidity after major hepatic resection for liver hydatid cyst was 19\% which is similar to the other authors' reports. 
In conclusion, the major hepatic resection which is a radical procedure is a safe and effective option for treatment of liver hydatidosis and should be performed whenever possible when the entire segment or lobe is diffusedly involved by one huge or multiple sized cysts with little healthy liver tissue left.

\section{REFERENCES}

[1] I. Sayek, R. Yalin and Y. Sanac, "Surgical Treatment of Hydatid Disease of the Liver," Archives of Surgery, Vol. 115, No. 7, 1980, pp. 847-850. doi:10.1001/archsurg.1980.01380070035007

[2] K. M. Harris, D. L. Morris, R. Tudor, P. Toghill and J. D. Hardcastte, "Clinical and Radiological Features of Simple and Hydatid Cysts of the Liver," British Journal of Surgery, Vol. 73, No. 10, 1986, pp. 835-838. doi: 10.1002/bjs. 1800731025

[3] W. Zhang, J. Li and D. P. McManus, "Concepts in Immunology and Diagnosis of Hydatid Disease," Clinical Microbiology Reviews, Vol. 16, No. 1, 2003, pp. 18-36. doi:10.1128/CMR.16.1.18-36.2003

[4] S. A. Barnes and K. D. Lillemoe, "Liver Abscess and Hydatid Cyst Disease,” In: M. J. Zinner, S. L. Schwartz and H. Eillis, Eds., Maingot's Abdominal Operation, Vol. II, 1977, pp. 1513-1545.

[5] W. S. Kammera and P. M. Schantz, "Ecchinococcal Disease," Infectious Disease Clinics of North America, Vol. 7, 1993, pp. 605-618.

[6] A. Flisser, "Larval Tapeworm Infections; Cysticercosis, Cystic Echinococcosis and Alveolar Echinococcosis," In: D. J. Richardson and P. J. Krause, Eds., Northamerica Parasitic Zoonoses, Kluwer Academic Publishers, Norwell, 2002, pp. 57-71.

[7] M. I. El Tahir, M. F. Omojole, T. Malatani, A. H. Alsaigh and O. A. Ogunbiyi, "Hydatid Disease of the Liver; Evaluation of Ultrasound and Computed Tomography," British Journal of Radiology, Vol. 65, 1992, pp. 390-392. doi:10.1259/0007-1285-65-773-390

[8] P. Mate Miranda, I. Osnaya Palma, U. Rodriguez Prado, A. Gutierrez Marin, M. Tawil, S. H. Gonzelez, et al., "Epidemiologic and Ultrasonographic Study of Echinococcosis in a Community in the State of Mexico," American Journal of Tropical Medicine and Hygiene, Vol. 77, No. 3, 2007, pp. 500-503.

[9] Z. Turkyilmaz, K. Sonmez, R. Karabulut, et al., "Conservative Surgery for Treatment of Hydatid Cysts in Children," World Journal of Surgery, Vol. 28, No. 6, 2004, pp. 597-601. doi:10.1007/s00268-004-7029-9

[10] I. Sayek, M. B. Timakeiz and R. Dogan, "Cystic Hydatid Disease: Current Trends in Diagnosis and Management," Surgery Today, Vol. 34, No. 12, 2004, pp. 987-996. doi:10.1007/s00595-004-2830-5

[11] P. K. Chowbey, S. Shah, R. Khullar, et al., "Minimal Access Surgery for Hydatid Cyst Disease: Laparocopic, Thoracoscopic, and Retroperitoneoscopic Approach," Journal of Laparoendoscopic \& Advanced Surgical Techniques, Vol. 13, No. 3, 2003, pp. 159-165.

\section{doi:10.1089/109264203766207672}

[12] H. Ustunsory, I. Akaemir, M. C. Sivrikoz, et al., "Cardiac Hydatid Cyst: Report of Two Cases," Heart, Lung and Circulation, Vol. 11, 2002, pp. 117-120.

[13] S. Saini, P. R. Mueller, J. I. Ferrucci, et al., "Percutaneous Aspiration of Hepatic Cysts Does Not Provide Definitive Therapy," American Journal of Roentgenology, Vol. 141, No. 3, 1983, pp. 559-560.

[14] N. C. Armitage and L. H. Blummgarten, "Partial Resection and Fenestration in the Treatment of Polycystic Liver Disease," British Journal of Surgery, Vol. 71, No. 3, 1984, pp. 242-244. doi:10.1002/bjs.1800710331

[15] S. Iwatsuki, D. G. Sheahan and T. E. Starzl, "The Changing Face of Hepatic Resection," Current Problems in Surgery, Vol. 26, No. 5, 1989, pp. 289-379. doi:10.1016/0011-3840(89)90022-1

[16] S. Iwatsuki, S. Todo and T. E. Starzl, "Excisional Therapy for Benign Hepatic Lesions," Surgery, Gynecology \& Obstetrics, Vol. 171, No. 3, 1990, pp. 240-246.

[17] D. Voros, D. Katsarelias, G. Polymoneas, A. Polydorou, L. Pisitiolis, A. Kalovidouris, et al., "Treatment of Hydatid Liver Disease," Surgical Infections, Vol. 8, No. 6, 2007, pp. 621-627. doi:10.1089/sur.2006.0070

[18] H. A. Gharbi, W. Hessine, M. W. Brauner and K. Dupuch, "Ultrasound Examination of Hydatid Liver," Radiology, Vol. 139, 1981, pp. 459-463.

[19] F. Guiliant, F. D'Acapito, M. Vellone, et al., "Risk for Laparoscopic Fenestration of Liver Cysts," Surgical Endoscopy and Other Interventional Techniques, Vol. 17, No. 11, 2003, pp. 1735-1738. doi:10.1007/s00464-002-9106-1

[20] A. M. El Shazly, S. E. Awad, M. A. Hegazy, K. A. Mohammad and T. A. Morsy, "Echinococcosis Granulosus Hydatidosis an Endemic Zoonotic Disease in Egypt," Journal of the Egyptian Society of Parasitology, Vol. 37, No. 2, 2007, pp. 609-622.

[21] H. M. Salama, N. H. Ahmed, N. Eldeeb and R. Ahmed, "Hepatic Hydatid Cysts: Sonographic Follow Up after Percutaneous Sonographically Guided Aspiration," Journal of Clinical Ultrasound, Vol. 26, No. 9, 1998, pp. 455460.

doi:10.1002/(SICI)1097-0096(199811/12)26:9<455::AIDJCU4>3.0.CO;2-M

[22] I. Sayek and D. Onat, "Diagnosis and Treatment of Uncomplicated Hydatid Cyst of the Liver," World Journal of Surgery, Vol. 25, No. 1, 2001, pp. 21-27. doi: $10.1007 / \mathrm{s} 002680020004$

[23] A. Chiotis, A. Tsaroucha, A. Ioannidis, et al., "Use of an Aspiration Apparatus in the Surgical Treatment of Echinoccal Cysts," Chirurgia, Vol. 16, 2003, pp. 5-7.

[24] A. Zaouche, K. Haouet, M. Jouini, A. El hachaichi and C. Dziri, "Management of Liver Hydatid Cysts with a Large Biliocyst Fistula: Multicenter Retrospective Study," World Journal of Surgery, Vol. 25, No. 1, 2001, pp. 28-39. doi:10.1007/s002680020005

[25] S. Demircis, S. Eraslan, E. Anadol and L. Bozatli, "Comparison in the Results of Different Surgical Techniques in the Management of Hydatid Cysts of the Liver," World 
Journal of Surgery, Vol. 13, No. 1, 1989, pp. 88-91. doi:10.1007/BF01671161

[26] S. Marani, G. Canossi, F. Nicoli, G. Alberti, S. Maoni and P. Casolo, "Hydatid Disease: MRI Imaging Study," Radiology, Vol. 175, No. 3, 1990, pp. 701-706.

[27] E. Ozaslan and Y. Bayrakter, "Endoscopic Therapy in the Management of Hepatobiliary Hydatid Disease," Journal of Clinical Gastroenterology, Vol. 35, No. 2, 2002, pp. 160-174. doi:10.1097/00004836-200208000-00009

[28] H. Simsek, E. Ozaslan, I. Sayek, C. Svas, O. Abasoglu, A. Soylu, et al., "Diagnosis and Therapeutic ERCP in Hepatic Disease," Gastrointestinal Endosc, Vol. 58, No. 3, 2003, pp. 384-389. doi:10.1067/S0016-5107(03)00013-0

[29] A. Davis, Z. S. Pawlowski and H. Dixon, "Multicentre Clinical Trial of Bemantazle Carbamates in Human Echinococcosis," Bulletin of the World Health Organization, Vol. 64, 1986, pp. 383-388.

[30] A. Bilge and E. M. Sozuer, "Diagnosis and Surgical Treatment of Hepatic Hydatid Disease," HPB Surgery, Vol. 6, No. 1, 1992, pp. 57-64. doi:10.1155/1992/41956

[31] S. Berrada, B. Essadki and N. Zerouali, "Kyste Hydatique du Foie. Treatment par Resection du Dome Saillant. Notre Experience a Propos d'Une Serie de 495 Cases," Annales De Chirurgie, Vol. 47, 1993, pp. 510-512.

[32] C. Kayaalp, B. Bostana, S. Yol and M. Akoglu, "Distribution of Hydatid Cysts into the Liver with Reference to Cystobiliary Communications and Cavity-Related Complications," American Journal of Surgery, Vol. 185, No. 2, 2003, pp. 175-179. doi:10.1016/S0002-9610(02)01202-3

[33] A. Alper, O. Ariogul, A. Emre, A. Uras and A. Okten, "Choledochoduodenostomy for Intrabiliary Rupture of Hydatid Cysts of Liver," British Journal of Surgery, Vol. 74, No. 4, 1987, pp. 243-245. doi:10.1002/bjs. 1800740405

[34] O. Elbir, H. Gundoghu, M. Gagilulekci, C. Kayaalp, F. Atalay, M. Savkilioglu, et al., "Surgical Treatment of Intrabiliary Rupture of Hydatid Cysts of the Liver: Comparison of Choldochoduodenestomy with T-Tube Drainage," Digestive Surgery, Vol. 18, No. 4, 2001, pp. 289293. doi: $10.1159 / 000050154$

[35] K. Yorganci and I. Sayek, "Surgical Treatment of Hydatid Cysts of the Liver in the Era of Percutaneous Treatment," American Journal of Surgery, Vol. 184, No. 1, 2002, pp. 63-69. doi:10.1016/S0002-9610(02)00877-2

[36] WHO/OJE, "Manual on Echinoccosis in Humans and Animals. A Public Health Problem of Global Concern," World Organization, Paris, 2001.

[37] C. S. Ramachandran, D. Goel and V. Arora, "Laparoscopic Surgery in Hepatic Hydatid Cysts: A Technical
Improvement," Surgical Laparoscopy, Endoscopy \& Percutaneous Techniques, Vol. 11, No. 1, 2001, pp. 14-18. doi:10.1097/00129689-200102000-00004

[38] R. Adam, A. Laurent, D. Aizoulay, D. Castaring and H. Bismuth, "Two-Satge Hepatectomy: A Planned Strategy to Treat Irresectable Liver Tumors," Annals of Surgery, Vol. 232, No. 6, 2000, pp. 777-785. doi:10.1097/00000658-200012000-00006

[39] J. M. Little, M. J. Hallands and H. Ekberg, "Recurrence of Hydatid Disease of the Liver," World Journal of Surgery, Vol. 12, No. 5, 1988, pp. 700-704. doi:10.1007/BF01655892

[40] C. Dziri, K. Haouet and A. Fingerhut, "Treatment of Hydatid Cyst of the Liver: Where Is the Evidence?" World Journal of Surgery, Vol. 28, No. 8, 2004, pp. 731-736. doi:10.1007/s00268-004-7516-Z

[41] S. Demircis, S. Eraslan, E. Anadol and L. Bozatli, "Comparison of the Results of Different Surgical Techniques in the Management of Hydatid Cysts of the Liver," World Journal of Surgery, Vol. 13, No. 1, 1989, pp. 88-91. doi:10.1007/BF01671161

[42] P. Magistrelli, R. Masetti, R. Coppola, A. Messia, G. Nuzzo and A. Picciocchi, "Surgical Treatment of Hydatid Disease of the Liver," Archives of Surgery, Vol. 126, No. 4, 1991, pp. 518-523. doi:10.1001/archsurg.1991.01410280122020

[43] A. A. Balik, M. Basoglu, F. Celebi, D. Oren, K. Y. Palat, S. S. Atamanalp, et al., "Surgical Treatment of Hydatid Disease of the Liver: Review of 304 Cases," Archives of Surgery, Vol. 134, No. 2, 1999, pp. 166-169. doi:10.1001/archsurg.134.2.166

[44] R. Assadourian, G. Leynaud and N. Atie, "Traitment du Cystehydatique du Foie: Notre Attitade Actuelle," Journal De Chirurgie, Vol. 117, 1980, pp. 115-120.

[45] D. D. Karavias, C. E. Vaglanos, N. Bouboulis, et al., "Improved Techniques in the Surgical Treatment of Hepatic Hydatidosis," Surgery, Gynecology \& Obstetrics, Vol. 174, No. 3, 1992, pp. 176-180.

[46] F. S. Rakas, M. Elmufti, P. M. Mehta, et al., "Omentoplasty or Tube Drainage for the Management of the Residual Cavity Following the Removal of Hepatic Hydatid cysts," Hepatogastroeneterolgy, Vol. 37, Suppl. 2, 1990, pp. 55-57.

[47] C. Dziri, J. C. Paquet, J. M. Hay, et al., "Omentoplasty in the Prevention of Deep Abdominal Complications after Surgery for Hydatid Disease of the Liver: A Multicenter Prospective Randomized Trial," Journal of the American College of Surgeons, Vol. 188, No. 3, 1999, pp. 281-289. doi:10.1016/S1072-7515(98)00286-5 